\title{
Random Expected Utility and Certainty Equivalents: \\ Mimicry of Probability Weighting Functions
}

\author{
Nathaniel T. Wilcox \\ Economic Science Institute \\ Chapman University \\ Orange, CA 92866 \\ nwilcox@chapman.edu
}

October 25, 2017

\begin{abstract}
For simple prospects routinely used for certainty equivalent elicitation, random expected utility preferences imply a conditional expectation function that can mimic deterministic rank dependent preferences. That is, a subject with random expected utility preferences can have expected certainty equivalents exactly like those predicted by rank dependent probability weighting functions of the inverse-s shape discussed by Quiggin (1982) and advocated by Tversky and Kahneman (1992), Prelec (1998) and other scholars. Certainty equivalents may not nonparametrically identify preferences: Their conditional expectation (and critically, their interpretation) depends on assumptions concerning the source of their variability.
\end{abstract}

Keywords: Certainty Equivalence, Identification, Preference Estimation, Preference Measurement, Random Preference, Choice Under Risk and Uncertainty

JEL Codes: C81, C91, D81

I am grateful to the Economic Science Institute and Chapman University for their ongoing support. Jose Apesteguia, Mattias Del Campo Axelrod, Miguel A. Ballester, Sudeep Bhatia, Michael Birnbaum, Daniel Cavagnaro, Mark DeSantis, Glenn W. Harrison, Anthony Marley, Kevin Mumford, John P. Nolan and Mark Schneider provided help or commentary, though none are responsible for remaining errors. 
Elicitation of certainty equivalents has become routine in laboratory measurement of preferences under risk and uncertainty (Tversky and Kahneman 1992; Tversky and Fox 1995; Wu and Gonzales 1999; Gonzales and Wu 1999; Abdellaoui 2000; Abdellaoui, Bleichrodt and Paraschiv 2007; Halevy 2007; Bruhin, Fehr-Duda and Epper 2010; Vieider et al. 2015). While elicitation methods vary across such studies, formal empirical interpretations of elicited certainty equivalents are invariably the same. The subject is assumed to have a unique and fixed preference order, implying (under unchanged conditions of background wealth, risk and so forth) a unique and fixed certainty equivalent for each prospect. Elicited certainty equivalents are then interpreted as this unique and fixed certainty equivalent plus some error of banal origin with standard properties.

Such added error, or something like it, is necessary: In repeated elicitations using exactly the same prospect, elicited certainty equivalents vary within subjects (Tversky and Kahneman 1992, p. 306-308; Krahnen, Rieck and Theissen 1997, p. 477; von Winterfeldt et al. 1997, p. 422; Gonzalez and Wu 1999, pp. 144-146; Pennings and Smidts 2000, p. 1342) and other evidence also suggests inherent variability of elicited certainty equivalents (e.g. Butler and Loomes 2007). Luce (1997, pp. 81-82) argued that theory and empirical interpretation need to take a position on such response variability. Adding mean zero error to an otherwise deterministic model of certainty equivalents is clearly one option here, and I call this the standard model of an elicited certainty equivalent.

Random preference models are a well-known alternative to standard models. These models assume that an individual subject's preference order is a random variable, and that each certainty equivalent elicited from that subject is fully determined by a single realization of that random variable. Random preference models are both old and contemporary, particularly in the realm of discrete choice (Becker, DeGroot and Marschak 1963; Eliashberg and Hauser 1985; Hilton 1989; Loomes and Sugden 1995, 1998; Regenwetter and Marley 2001; Gul and Pesendorfer 2006; Regenwetter, Dana and DavisStober 2011; Ahn and Sarver 2013; Apesteguia and Ballester 2016; Karni and Safra 2016).

I examine implications of random preference models for elicited certainty equivalents and find a significant complication of their empirical interpretation. Random model expected utility preferences (or more simply random EU as Gul and Pesendorfer call it) imply expected certainty equivalents that can mimic those implied by standard model rank- 
dependent preferences (or more simply standard $R D U$ ). That is, a random EU subject can have expected certainty equivalents that appear to reveal rank dependent probability weighting functions of the inverse-s shape discussed by Quiggin (1982) and advocated by Tversky and Kahneman (1992) and other scholars.

Section 1 develops a standard RDU model and a random EU model of observed certainty equivalents in formal econometric terms, separating both into a conditional expectation function and an error term. Section 2 then develops parametric examples of the random EU model that mimic standard RDU models: For example, Prelec (1998) weighting functions will be derived from certainty equivalents governed by a specific random EU model. Section 3 shows that a class of random EU models will display apparent underweighting of high probabilities and apparent overweighting of low probabilities-just that pattern implied by inverse-s probability weighting functions in a standard RDU model; and Section 4 provides brief graphical intuition for all the results. Contra widespread suggestions to the contrary, I conclude that elicited certainty equivalents may not nonparametrically identify preferences, since their conditional expectation (and critically, the interpretation of it) depends on the source of their variability.

\section{Certainty equivalents of simple prospects under Standard RDU and Random EU}

Simple prospects $(W, p)$ deliver an outcome $W>0$ with probability $p$ and nothing with probability $1-p$. Many scholars believe that observed certainty equivalents of simple prospects reveal the probability weighting function of the rank-dependent preference family when the utility or value of outcomes is linear or nearly so (e.g. Tversky and Kahneman 1992; Prelec 1998). To see this, let the utility or value of outcomes $z$ have the power form $v(z)=z^{1 / x}$ where $x \in(0, \infty)$ : I write the power as $1 / x$ for later convenience. The rank dependent utility or $\operatorname{RDU}$ of $(W, p)$ is then $\pi(p \mid \omega) \cdot W^{1 / x}$, where $\pi(p \mid \omega)$ is a probability weighting function depending on preference parameters $\omega$. The certainty equivalent of $(W, p)$ is then $\pi(p \mid \omega)^{x} \cdot W$, but one commonly divides this by $W$ to free it of dependence on $W$ and calls $C^{r d}(p \mid x, \omega) \equiv \pi(p \mid \omega)^{x}$ the RDU relative certainty equivalent of a simple prospect. Notice that when $x=1$ (i.e. for a linear value of outcomes), one has 
$C^{r d}(p \mid 1, \omega) \equiv \pi(p \mid \omega)$, so relative certainty equivalents of simple prospects are thought to reveal RDU (or CPT) probability weighting functions when $x=1$. Expected utility or EU is the special case where $\pi(p \mid \omega) \equiv p$, so also define $C^{e u}(p \mid x) \equiv p^{x}$ as the EU relative certainty equivalent of any simple prospect (given specific $x$ ).

Let $c e$ be the observed certainty equivalent for $(W, p)$ elicited from a subject, and let $c=c e / W \in[0,1]$ be the observed relative certainty equivalent. $E(c \mid p)$ is the expected value of $c$ given the win probability $p$ : Many econometricians call this the conditional expectation function or c.e.f. of $c$. Defining an error as $\varepsilon=c-E(c \mid p)$, rearrangement gives a general additive empirical specification for observed relative certainty equivalents, which is $c=E(c \mid p)+\varepsilon$. A standard RDU model then assumes that the c.e.f. $E(c \mid p)$ is the theoretical one given by RDU, that is $C^{r d}(p \mid x, \omega) \equiv \pi(p \mid \omega)^{x}$, yielding the specification $c=\pi(p \mid \omega)^{x}+\varepsilon$. Various estimations of $(x, \omega)$ may then proceed: Bruhin, Fehr-Duda and Epper (2010) use maximum likelihood, while Tversky and Kahneman (1992) use nonlinear least squares. In this model the error term $\varepsilon$ is thought to arise from banal sources such as "carelessness, hurrying, or inattentiveness" (Bruhin, Fehr-Duda and Epper p. 1383).

Before continuing to the specification of a random EU model, note the following important fact about standard RDU models of certainty equivalents. For many weighting functions $\pi(p \mid \omega), x$ and $\omega$ cannot be wholly identified solely from the relative certainty equivalents of simple prospects. For instance suppose $\pi(p \mid \omega)$ is the 2-parameter Prelec (1998) weighting function $\exp \left(-\beta[-\ln (p)]^{\alpha}\right)$ where $\alpha$ and $\beta$ are strictly positive parameters. Relative certainty equivalents of simple prospects will then be $C^{r d}(p \mid x, \alpha, \beta)=$ $\exp \left(-x \beta[-\ln (p)]^{\alpha}\right)$, so clearly only $\alpha$ and the product $x \beta$ can be estimated from simple prospects alone. Scholars know this quite well, so experimental designs meant to separately estimate all three parameters from certainty equivalents must (and typically do) contain at least some non-simple prospects (those containing at least two possible nonzero outcomes). I focus on simple prospects because of their tractability and their simple interpretation under standard RDU: $C^{r d}(p \mid 1, \omega) \equiv \pi(p \mid \omega)$, so relative certainty equivalents of simple prospects reveal weighting functions at linear $v(z)$ (Tversky and Kahneman 1992; Prelec 1998). The Monte Carlo study in my appendix employs an experimental 
design (that of Gonzalez and Wu 1999) containing both simple and non-simple prospects, and results of that study will echo the analytical results I show here for simple prospects.

In the general RDU case, a random preference model could take both $x$ and $\omega$ to be realizations of nondegenerate random variables $X$ and $\Omega$ within a subject. However, existing random preference estimations (e.g. Loomes, Moffatt and Sugden 2002; Wilcox 2008,2011 ) treat weighting function parameters $\omega$ as fixed within a subject, and contemporary random preference theory seems to be confined to treatment of $X$ as random only (e.g. Gul and Pesendorfer 2006; Apesteguia and Ballester 2016). Therefore, all of my random preference analysis treats only $x$ as the realization of a random variable $X$; and in any case, my random model analysis is mostly confined to the random EU case.

In any distinct elicitation trial, an independent random EU model, or simply random EU for short, assumes that an independent and identically distributed realization $x$ of $X$ occurs and fully determines the relative certainty equivalent $C^{e u}(p \mid x)=p^{x}$. Assume that a probability density function $f(x \mid \psi)$ of $X$ with support $(0, \infty)$ lies within a subject, with parameters $\psi$ governing moments, location and/or scale. Then define $\mathbb{C}^{e u}(p \mid \psi)$ as

$$
\mathbb{C}^{e u}(p \mid \psi) \equiv E_{X}\left[C^{e u}(p \mid x)\right]=\int_{0}^{\infty} p^{x} f(x \mid \psi) d x=\int_{0}^{\infty} \exp (-x \tau) f(x \mid \psi) d x
$$

The final integral in eq. 1 (which becomes useful later in Section 2) simply defines $\tau=-\ln (p)$ and rewrites $p^{x}$ as $\exp (-x \tau)$. The function $\mathbb{C}^{e u}(p \mid \psi)$ is the expected relative certainty equivalent of a random EU subject for simple prospects $(W, p)$, given her underlying p.d.f. $f(x \mid \psi)$. We will have an empirical model $c=E(c \mid p)+\xi$ of the same form as the standard RDU model. However, the random EU c.e.f. is $E(c \mid p)=\mathbb{C}^{e u}(p \mid \psi)$ as given by eq. 1 , and the error $\xi$ is simply defined as $p^{x}-\mathbb{C}^{e u}(p \mid \psi)$. The eq. 1 definition implies that these new errors $\xi$ also satisfy the usual properties $(E(\xi)=E(\xi \mid p)=0)$, so we have a close resemblance between the random EU model $c=\mathbb{C}^{e u}(p \mid \psi)+\xi$ and the standard RDU model $c=C^{r d}(p \mid x, \omega)+\varepsilon$ and can estimate both using the same variety of estimators.

To conclude this section, allow a brief digression on elicitation methods. There is another way of thinking about the p.d.f. $f(x \mid \psi)$ of $X$ in the Random EU model. Suppose an experimenter uses some method $M$ to elicit certainty equivalents $c e$ from a subject, figures 
the relative certainty equivalents $c=c e / W$, and computes $x(c, p)$ to solve $p^{x}=c$; that is, let $x(c, p) \equiv \ln (c) / \ln (p)$. Suppose that in repeated elicitations using method $M$, across various values of $p$, the empirical c.d.f. of $x(c, p)$ is observed to be $\widehat{F}_{M}(x \mid p)$ which converges to $F_{M}(x \mid p)$ as the sample of observations grows. If $F_{M}(x \mid p)$ is in fact independent of $p$ and so just $F_{M}(x)$, the variability of the relative certainty equivalents observed by the experimenter could be interpreted as arising from a random EU model of the kind assumed here, where a p.d.f. $f_{M}(x \mid \psi)$ is derived from $F_{M}(x)$. This suggests ways in which one might test versions of the random EU model (or versions of a random RDU model, and later I will return to this), but also shows that the results here only require that elicitation methods satisfy two key assumptions: (1) repeated trials using the method yield variability in elicited certainty equivalents; and (2) this variability is consistent with the assumptions of a random EU model-namely, that $F_{M}(x)$ is independent of $p$. Neither assumption rules out dependence of $f(x \mid \psi)$ on the elicitation method $M$; and debates about the relative merits of various elicitation methods need not impinge on these assumptions in any necessary way.

\section{Parametric examples of random EU mimicry of standard RDU}

The close resemblance between the standard RDU and random EU models suggests two possible types of mimicry. First, since $C^{r d}(p \mid 1, \omega) \equiv \pi(p \mid \omega)$ in standard RDU, it will be troubling if $\mathbb{C}^{e u}(p \mid \psi)$ can "look like" a stereotypical $\pi(p \mid \omega)$, that is, can have properties like those that many scholars believe are empirically characteristic of RDU weighting functions. I will refer to this as weak mimicry (of standard RDU by random EU). Second, it may happen that for some well-known and specific $\pi(p \mid \omega)$, there exists a specific $f(x \mid \psi)$ such that $\mathbb{C}^{e u}(p \mid \psi)$ is a re-parameterization of $C^{r d}(p \mid x, \omega)$. To formally define what that means, let $D$ be the set of possible parameter vectors $(x, \omega)$, and let $\Psi$ be the set of possible parameter vectors $\psi$. Then suppose that, for some specific $f(x \mid \psi)$, there exists a function $H_{f}: D \rightarrow \Psi$ such that $\mathbb{C}^{e u}\left[p \mid H_{f}(x, \omega)\right] \equiv C^{r d}(p \mid x, \omega) \equiv \pi(p \mid \omega)^{x}$ : Then one may say there is strong mimicry (of standard RDU by random EU) for $f(x \mid \psi)$. Notice that strong mimicry implies weak mimicry but not vice versa. 
Since $-\ln (p)>0 \forall p \in(0,1)$, so that $\tau>0$ too, the final integral in eq. 1 is the onesided Laplace transform $\mathcal{L}\{f\}(\tau)$ of the p.d.f. $f(x \mid \psi)$-provided it exists; and below I only use p.d.f.s for which the existence and form of $\mathcal{L}\{f\}(\tau)$ have been demonstrated and derived by others. In such instances, these known Laplace transforms $\mathcal{L}\{f\}(\tau)$ of a p.d.f. $f(x \mid \psi)$ make it simple to derive various parametric examples of $\mathbb{C}^{e u}(p \mid \psi)$, using the relationship $\mathbb{C}^{e u}(p \mid \psi)=\mathcal{L}\{f\}[-\ln (p)]$. Two examples follow.

Example 1. Suppose $X$ has the Gamma p.d.f.

$$
f(x \mid k, \theta)=\frac{1}{\Gamma(k) \theta^{k}} x^{k-1} \exp (-x / \theta) \text { for } x, k \text {, and } \theta \in(0, \infty),
$$

where $\Gamma$ is the Gamma function.

It's widely known that this has the Laplace transform $\mathcal{L}\{f\}(\tau)=(1+\theta \tau)^{-k}$, implying that

$$
\mathbb{C}^{e u}(p \mid k, \theta)=(1-\theta \ln (p))^{-k} .
$$

Figure 1-A shows this Gamma c.e.f. for $k=0.75$ and $\theta=2.79$. At these parameter choices, it has the "inverse-s" shape many believe is characteristic of weighting functions $\pi(p \mid \omega)$ and the fixed point $p \approx e^{-1}$ which is characteristic of Prelec's (1998) 1-parameter weighting function; so this is an instance of weak mimicry. One needs to say that this Gamma c.e.f. can (not must) weakly mimic this characteristic shape. Figure 1-B shows the Gamma c.e.f. for $k=0.75$ and $\theta=0.9$ : Here we see the "optimist" shape discussed by Quiggin (1982), and also the plurality shape of individually estimated weighting functions in Wilcox (2015). Such shape flexibility is also characteristic of 2-parameter weighting functions found in the literature on RDU and CPT estimation where such flexibility is usually regarded as a strength rather than a weakness (for estimation).

Similar examples may be given for any other parametric distribution with a Laplace transform, but the next specific example leads to a particularly interesting result.

Example 2. Suppose $X$ has the (unshifted) Lévy p.d.f.

$$
f(x \mid \lambda)=\frac{\lambda}{2 \sqrt{\pi}} x^{-3 / 2} \exp \left(\frac{-\lambda^{2}}{4 x}\right) \text { for } x \text { and } \lambda \in(0, \infty) .
$$


Figure 1-A. An Inverse-s Shaped Gamma Conditional Expectation Function

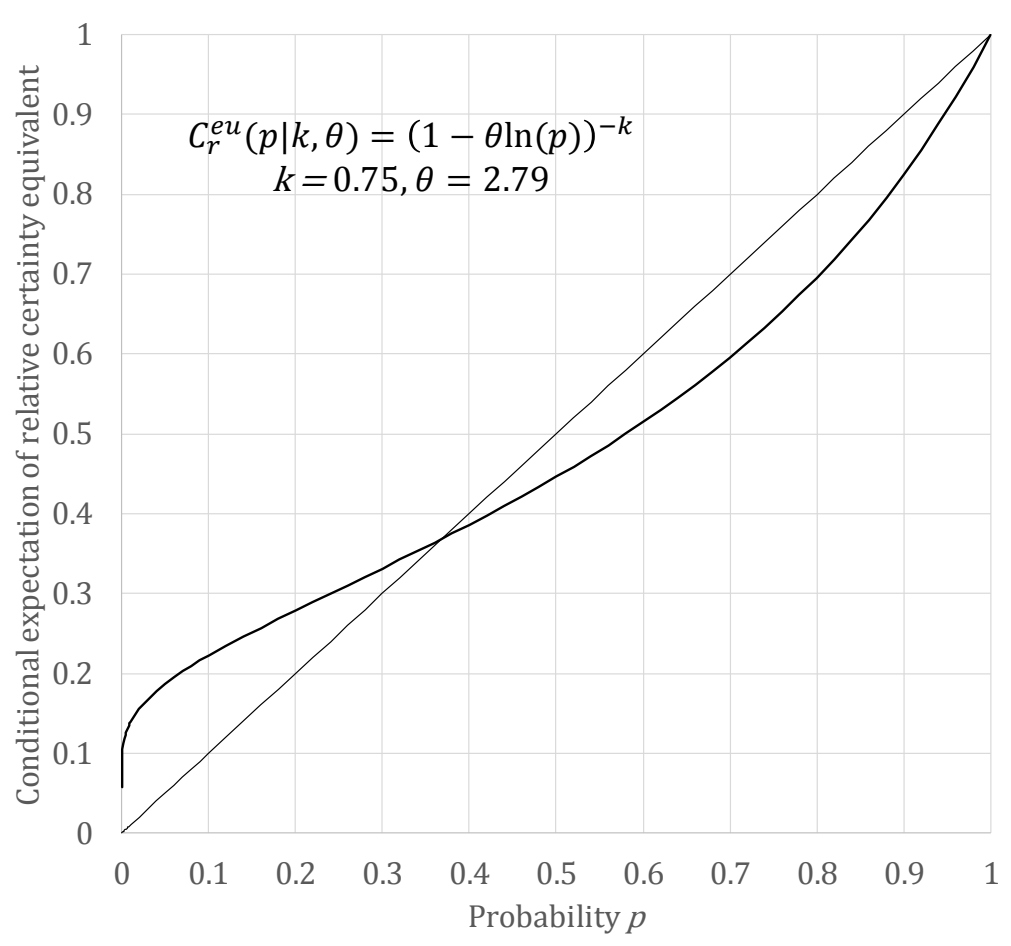

Figure 1-B. An "Optimist" Shaped Gamma Conditional Expectation Function

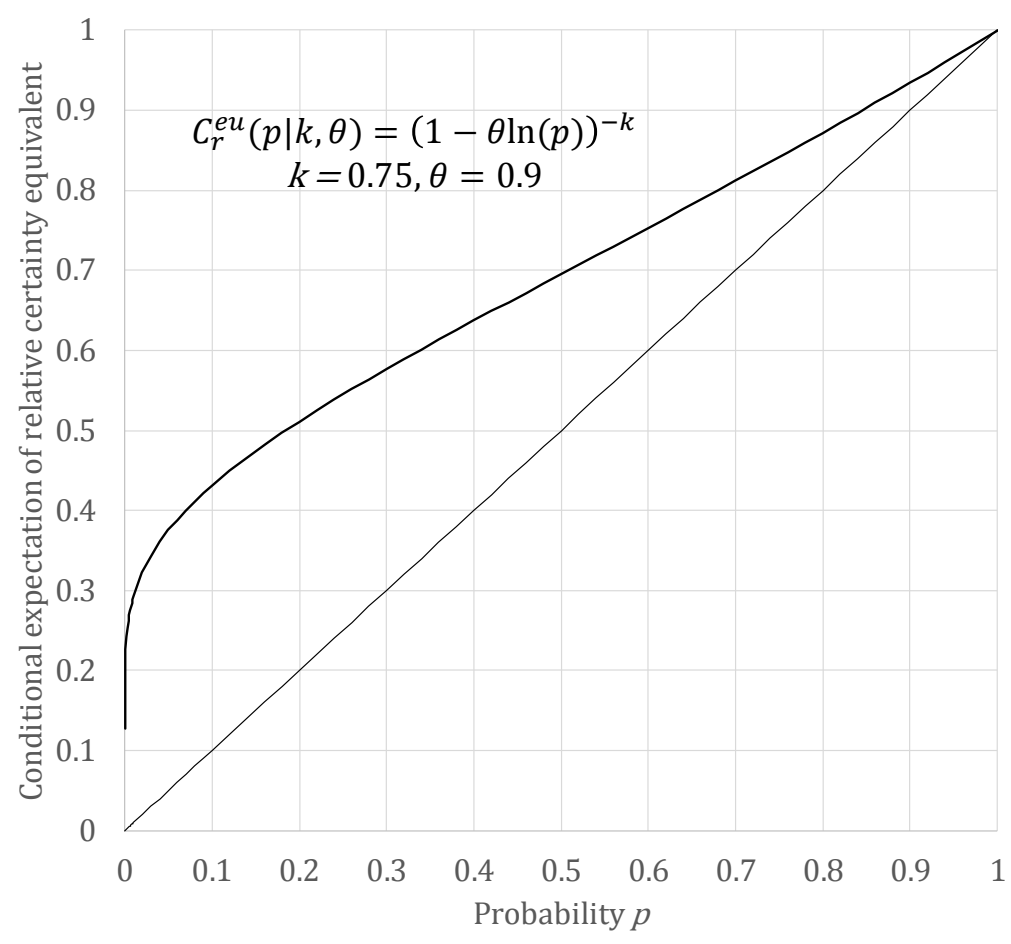


This has the Laplace transform $\mathcal{L}\{f\}(\tau)=\exp \left(-\lambda \tau^{1 / 2}\right)$ (González-Velasco 1995, p. 537), implying that

$$
\mathbb{C}^{e u}(p \mid \delta)=\exp \left(-\lambda[-\ln (p)]^{1 / 2}\right)
$$

Earlier I noted that in the case of the Prelec (1998) 2-parameter weighting function, $C^{r d}(p \mid x, \alpha, \beta)=\exp \left(-x \beta[-\ln (p)]^{\alpha}\right)$. Clearly, this is identical to eq. 3 if we set $\lambda=x \beta$ and require that $\alpha=1 / 2$. This is very close to being a case of strong mimicry, but not quite, since eq. 3 can only mimic Prelec weighting functions when $\alpha$ just happens to be $1 / 2$. Empirically, estimates of $\alpha$ have a wider range than a small neighborhood of $1 / 2$.

However, this result provides a strong and fruitful hint. The Lévy distribution is a specific instance of the Lévy Alpha-Stable distributions, also known simply as the Stable distributions. Except for special cases (Normal, Cauchy and Lévy), Stable random variables $X$ have no p.d.f. expressible in terms of elementary functions. However, their Laplace transforms exist as relatively simple expressions. For Stable random variables with support $(0, \infty)$, Nolan $(2018$, p. 109) shows that the Laplace transform exists and is $\mathcal{L}\{f\}(\tau)=$ $\exp \left\{-\gamma^{\delta}\left(\sec \frac{\delta \pi}{2}\right) \tau^{\delta}\right\}$, where $\gamma>0$ is a scale parameter and $\delta \in(0,1)$ is called the index of stability or characteristic exponent (see Feller 1971 and Hougaard 1986 for similar forms parameterized differently). Therefore, for Stable distributions of $X$ on $(0, \infty)$, we have

$$
\mathbb{C}^{e u}(p \mid \delta, \gamma)=\exp \left\{-\gamma^{\delta}\left(\sec \frac{\delta \pi}{2}\right)[-\ln (p)]^{\delta}\right\}
$$

Eq. 4 is identical to $C^{r d}(p \mid x, \alpha, \beta)=\exp \left(-x \beta[-\ln (p)]^{\alpha}\right)$ when we set $\delta=\alpha$ and set $\gamma=\left[\beta x\left(\cos \frac{\alpha \pi}{2}\right)\right]^{1 / \alpha}$, so a random EU model based on a Stable distribution of $X$ strongly mimics standard RDU with the 2-parameter Prelec (1998) function-provided that $\alpha<1$. Since this is both characteristic of most empirical estimates of $\alpha$ and indeed yields the characteristic inverse-s shape, this is strong mimicry of a well-known and widely used probability weighting function in the relevant part of the parameter space. 


\section{A general result}

A general result can be proved for a class of random EU models. Return to the more usual way of writing the power utility or value function, specifically $v(z)=z^{\sigma}$, thinking now of the power $\sigma$ as having some probability distribution within the subject, and make the following two assumptions.

Assumption One. The subject's distribution of $\sigma$ is non-degenerate and has a bounded support $\Sigma \subset(0, \infty)$. Specifically, $\underline{\sigma}=\inf (\Sigma)>0$ and $\bar{\sigma}=\sup (\Sigma)$ is finite.

Assumption Two. The expected value of $\sigma$ exists and is unity, that is $E(\sigma)=1$.

Assumption Two is meant to reflect a frequent (but by no means universal) finding when standard RDU or standard CPT models are estimated from certainty equivalents, namely, that estimated utility or value functions $v(z)$ are very nearly linear or at best very mildly concave (see e.g. Tversky and Kahneman 1992; Bruhin, Fehr-Duda and Epper 2010).

Under Expected Utility, the relative certainty equivalent of a simple prospect (given any value of $\sigma$ ) will be $p^{1 / \sigma}$, whose second derivative with respect to $\sigma$ is

$$
\frac{\partial^{2}}{\partial \sigma^{2}} p^{1 / \sigma}=\frac{-\ln (p) p^{1 / \sigma}}{\sigma^{4}}[-\ln (p)-2 \sigma]>0 \text { for all } p<\exp (-2 \sigma) .
$$

Define $\underline{p}=\exp (-2 \bar{\sigma})$. By Assumption One, $0<\underline{p}<\exp (-2 \sigma) \forall \sigma \in \Sigma$. Therefore, eq. 5 shows that for any $p<\underline{p}, p^{1 / \sigma}$ is strictly convex in $\sigma \forall \sigma \in \Sigma$. Jensen's Inequality then implies that $E\left(p^{1 / \sigma}\right)>p^{1 / E(\sigma)}$. Since $E(\sigma)=1$ by Assumption Two, we have $E\left(p^{1 / \sigma}\right)>p$ for sufficiently small $p$. That is: If $\Sigma$ is bounded above and $E(\sigma)=1$ (the mean value function $v(z)$ is linear), mean relative certainty equivalents of simple prospects will exceed $p$ when $p$ is low enough. We have apparent overweighting of low enough probabilities.

Similarly, define $\bar{p}=\exp (-2 \underline{\sigma})$. By Assumption One, $1>\bar{p}>\exp (-2 \sigma) \forall \sigma \in \Sigma$. Therefore, eq. 5 shows that for any $p>\bar{p}, p^{1 / \sigma}$ is strictly concave in $\sigma \forall \sigma \in \Sigma$. Jensen's 
Inequality then implies that $E\left(p^{1 / \sigma}\right)<p^{1 / E(\sigma)}$. Since $E(\sigma)=1$ by Assumption Two, we have $E\left(p^{1 / \sigma}\right)<p$ for sufficiently high $p$. That is: If $\Sigma$ is bounded below away from zero and $E(\sigma)=1$ (the mean value function $v(z)$ is linear), mean relative certainty equivalents of simple prospects will be less than $p$ when $p$ is high enough. We have apparent underweighting of high enough probabilities. The following proposition has been proved.

Proposition: Suppose that a subject's utility or value of money is $v(z)=z^{\sigma}$, that this subject's behavior is described by an independent random EU model where the subject's distribution of $\sigma$ satisfies Assumptions One and Two. Then:

(1) $\exists \underline{p}>0 \mid E\left(p^{1 / \sigma}\right)>p \forall p<\underline{p}$ (apparent overweighting of sufficiently low probabilities); and

(2) $\exists \bar{p}<1 \mid E\left(p^{1 / \sigma}\right)<p \forall p>\bar{p}$ (apparent underweighting of sufficiently high probabilities).

Notice that the assumptions behind the proposition are only sufficient conditions: The parametric examples of Section 2 involve p.d.f.s with support $(0, \infty)$ (neither bounded above nor bounded below away from zero, as Assumption One requires).

\section{Graphical intuition}

Figure 2 provides some graphical intuition behind . Assume that the random EU subject has a binomial distribution of $\sigma$ such that $E(\sigma)=1$ : Specifically she has $\sigma=1 / 3$ with probability $3 / 4$ and $\sigma=3$ with probability $1 / 4$. Figure 2 shows the function $p^{1 / \sigma}$ for $\sigma \in(0,3]$, given two values of $p$. The upper heavy curve is for $p=0.95$ and, as can be seen, this curve is overwhelmingly and strongly concave: In this case, $E\left[0.95^{(1 / \sigma)}\right]<0.95$, so this subject appears to underweight the high probability 0.95 . The lower heavy curve is for $p=0.05$ and, as can be seen, this curve is first convex and, for $\sigma$ beyond about 1.5, very gently concave: Here, $E\left[0.05^{(1 / \sigma)}\right]>0.05$, so this subject also appears to overweight the low probability 0.05 . This graphical intuition suggests why one may easily derive the characteristic inverse-s shape from many p.d.f.s $f(x \mid \psi)$ underlying a random EU model. 
Figure 2. Behavior of relative certainty equivalents as $\sigma$ varies, at low and high $p$

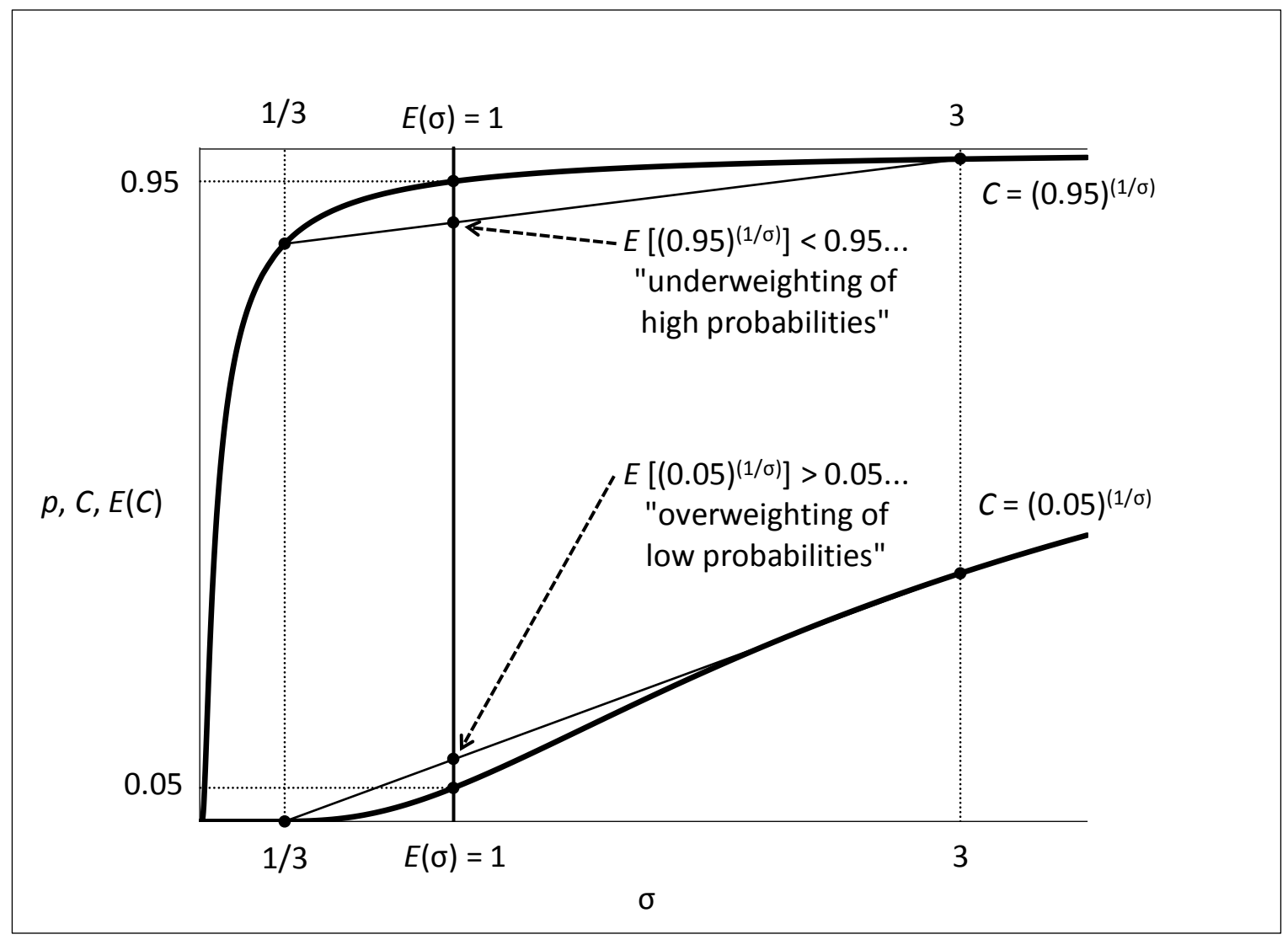

\section{Discussion and conclusions}

My results complicate interpretation of elicited certainty equivalents. However, I say 'complicate' rather than 'undermine' for several reasons. First, I have not shown that random preference EU and standard RDU certainty equivalents are indistinguishable. The formal results are entirely about conditional expectations and say nothing about conditional medians or conditional variances and other moments; and one might test both random EU and random RDU on the basis of these other characteristics.

For instance, recall that $C^{r d}(p \mid x, \omega) \equiv \pi(p \mid \omega)^{x}$ and suppose we now assume that $x$ is a realization of a random variable $X$, giving the simplest version of a random RDU model. Let $\mathrm{CV}$ denote coefficient of variation; then under random RDU, assuming that any weighting function parameters are fixed (not themselves random variables), we have 


$$
C V(-\ln (c)) \equiv \frac{\sqrt{V(-\ln (c))}}{E(-\ln (c))}=\frac{\sqrt{V(X)[-\ln (\pi(p \mid \omega))]^{2}}}{E(X)[-\ln (\pi(p \mid \omega))]}=\frac{\sqrt{V(X)}}{E(X)}=C V(X)
$$

This says that for any given subject, the coefficient of variation of $-\ln (c)$ will be equivalent to the coefficient of variation of $X$ and, moreover, independent of the particular $W$ and $p$ of any simple prospect $(W, p)$, regardless of whether the weighting function is an identity function (EU) or not (RDU). This immediately suggests a test of both random preference EU and RDU based on multiple (more than two) certainty equivalent elicitation trials for several different simple prospects. To my knowledge, such data are scarce but more could be gathered with appropriate experimental designs. The key point, however, is that for certainty equivalents, the random preference hypothesis can make strong refutable predictions about moments (here, a ratio of moments) that are independent of the form or even the presence of any rank-dependent weighting function.

Second, discrete choice experiments already suggest that random EU cannot be a complete model of discrete choices (e.g. Loomes and Sugden 1998). Under the random preference hypothesis, much of what EU predicts concerning pairs of related discrete choice problems remains unchanged relative to what EU predicts in its deterministic form (Loomes and Sugden 1995; Gul and Pesendorfer 2006; Wilcox 2008). This implies that many well-known discrete choice violations of EU also violate random EU. Here I showed once more (see Hilton 1989) that certainty equivalents are a different matter: Under random $\mathrm{EU}$, the expected values of certainty equivalents can mimic predictions of standard RDU and CPT. The upshot of this fact is that when one estimates risk models from certainty equivalents, part of the estimates (perhaps substantial parts) may reflect random preference heterogeneity as well as any underlying mean preference.

Third, my results only complicate estimation based on conditional expectation functions. While this is the overwhelmingly common basis for estimation, some of the empirical literature on RDU and CPT uses pooled sample conditional medians of certainty equivalents for description (Tversky and Kahneman 1992, pp. 309-311; Gonzalez and Wu 1999, p. 144-145). It may be that conditional median estimation (that is, least absolute deviation or LAD estimators) can solve the problem uncovered here. Recall the key role played by Jensen's Inequality in Section 3's general result: There is no counterpart of 
Jensen's Inequality for medians. No subject-level conditional median estimations (based on elicited certainty equivalents, using LAD estimation) of either RDU or CPT models are available. (Tversky and Kahneman 1992 do estimate a pooled sample weighting function from pooled sample conditional medians, but using a nonlinear least squares estimator.) My appendix looks at a LAD estimator and finds encouraging results for random EU data, but not for standard model EU data. I know of no estimator that correctly identifies weighting functions regardless of the true probabilistic model generating the data; finding such an estimator would be a nice contribution to decision research.

However, meaningful preference measurement may not be possible without strong assumptions concerning the random part of decision behavior (Wilcox 2008; Blavatskyy and Pogrebna 2010; Wilcox 2011; Apesteguia and Ballester 2016). Some say that elicited certainty equivalents permit "nonparametric" (or "parameter-free") identification and estimation of preferences (Gonzales and Wu 1999; Abdellaoui 2000; Bleichrodt and Pinto 2000; Abdellaoui, Bleichrodt and Paraschiv 2007) and many others repeat it (e.g. Prelec 1998; Luce 2000; Nielson 2003; Fox and Poldrack 2009; Wakker 2010). "Nonparametric" means many different things, but many econometricians divide discussion of models in two parts: (1) a conditional expectation function, or perhaps a conditional median function, and (2) the error, the random part that remains once such a function has been removed in a way that makes the expectation (or median) of the error zero. In the preference measurement literature, scholars who say their estimation is "nonparametric" (or "parameter-free") mean they are making few or no assumptions about the form of preference entities (utilities or values, and probability weights, and so forth) that appear in standard model conditional expectation functions. However, they routinely make the strong assumption of the standard model itself, and an old and well-developed alternative (the random preference model) has complicating consequences.

The essence of the standard model assumption is that the c.e.f. has an obvious interpretation - the intended interpretation being that of algebraic (deterministic) decision theory. Hendry and Morgan (2005, p. 23) argue that when we speak of model identification, we have things in mind beyond the original Cowles Foundation meaning-including "correspondence to the desired entity" and "satisfying the assumed interpretation (usually of a theory model)." Estimation of preferences from elicited certainty equivalents is 
complicated in just these senses. The standard model is but one probabilistic model assumption, and under a venerable and contemporary alternative-the random preference model-the c.e.f. in part reflects the underlying distribution of preferences within the subject, in ways that can mimic "the desired entity," the preference entity called the probability weighting function. I do not know whether certainty equivalents can nonparametrically identify such entities: This question needs a good answer. However, at this time there is certainly no rigorous reason to think elicited certainty equivalents free scholars of critical interpretive assumptions. As is true of discrete choices, it seems we choose a probabilistic model the moment we interpret certainty equivalents. 


\section{References}

Abdellaoui, M., 2000, Parameter-free elicitation of utility and probability weighting functions. Management Science 46, 1497-1512.

Abdellaoui, M., H. Bleichrodt and C. Paraschiv, 2007, Loss aversion under prospect theory: A parameter-free measurement. Management Science 53, 1659-1674.

Ahn, D. S. and T. Sarver, 2013, Preference for flexibility and random choice. Econometrica 81:341-361.

Apesteguia, and M. Ballester, 2016, Monotone stochastic choice models: The case of risk and time preferences. Journal of Political Economy (forthcoming).

Becker, G. M., M. H. DeGroot and J. Marschak, 1963, Stochastic models of choice behaviour. Behavioral Science 8, 41-55.

Blavatskyy, P. and G. Pogrebna, 2010, Models of stochastic choice and decision theories: Why both are important for analyzing decisions. Journal of Applied Econometrics 25, 963-986.

Bleichrodt, H. and J. L. Pinto, 2000, A parameter-free estimation of the probability weighting function in medical decision analysis. Management Science 46:1485-1496.

Bruhin, A., H. Fehr-Duda and T. Epper, 2010, Risk and rationality: Uncovering heterogeneity in probability distortion. Econometrica 78:1375-1412.

Butler, D. and G. Loomes, 2007, Imprecision as an account of the preference reversal phenomenon. American Economic Review 97, 277-297.

Eliashberg, J. and J. R. Hauser, 1985, A measurement error approach for modeling consumer risk preference. Management Science 31, 1-25.

Feller, W., 1971, An Introduction to Probability Theory and Its Applications Vol. 2, 2nd ed. New York: Wiley.

Fox, C. R. and R. A. Poldrack, 2009, Prospect theory and the brain. In P. Glimcher, C. Camerer, E. Fehr and R. Poldrack, eds., Neuroeconomics: Decision Making and the Brain. London, UK: Academic Press.

González-Velasco, E. A., 1995, Fourier analysis and boundary value problems. San Diego: Academic Press. 
Gonzalez, R. and G. Wu, 1999, On the shape of the probability weighting function. Cognitive Psychology 38, 129-166.

Gul, F. and W. Pesendorfer, 2006, Random expected utility. Econometrica 74,121-146.

Halevy, Y., 2007, Ellsberg revisited: An experimental study. Econometrica 75, pp. 503-536.

Hendry, D. F. and M. S. Morgan, 2005, The foundations of econometric analysis. Cambridge: Cambridge University Press.

Hilton, R.W., 1989. Risk attitude under random utility. Journal of Mathematical Psychology $33,206-222$.

Hougaard, P., 1986, Survival models for heterogeneous populations derived from stable distributions. Biometrika 73:387-396.

Karni, E. and Z. Safra, 2016, A theory of stochastic choice under uncertainty. Journal of Mathematical Economics 63:164-173.

Krahnen, J. P., C. Rieck and E. Theissen, 1997, Inferring risk attitudes from certainty equivalents: Some lessons from an experimental study. Journal of Economic Psychology 18:469-486.

Loomes, G., P. Moffatt, R. Sugden, 2002, A microeconometric test of alternative stochastic theories of risky choice. Journal of Risk and Uncertainty 24, 103-130.

Loomes, G. and R. Sugden, 1995, Incorporating a stochastic element into decision theories. European Economic Review 39, 641-648.

Loomes, G. and R. Sugden, 1998, Testing different stochastic specifications of risky choice. Economica 65, 581-598.

Luce, R. D., 1997, Some unresolved conceptual problems in mathematical psychology. Journal of Mathematical Psychology 41:79-87. ,2000, Utility of Gains and Losses: Measurement-Theoretical and Experimental Approaches. London, UK: Erlbaum.

Nelder, J. A. and R. Mead, 1965, “A simplex method for function minimization." Computer Journal 7, 308-313.

Nielson, W. S., 2003, Probability transformations in the study of behavior toward risk. Synthese 135:171-192.

Nolan, J. P., 2018 (forthcoming), Stable Distributions-Models for Heavy Tailed Data. Boston: Birkhauser. 
Pennings, J. M. E. and A. Smidts, 2000, Assessing the construct validity of risk attitude. Management Science 46:1337-1348.

Powell, M. J. D., 1992, A direct search optimization method that models the objective and constraint functions by linear interpolation. Technical Report DAMTP 1992/NA5, Department of Applied Mathematics and Theoretical Physics, University of Cambridge. Prelec, D., 1998, The probability weighting function. Econometrica 66, 497-527. Quiggin, J., 1982, A theory of anticipated utility. Journal of Economic Behavior and Organization 3, 323-343.

Regenwetter, M., J. Dana and C. P. Davis-Stober, 2011, Transitivity of preferences. Psychological Review 118:42-56.

Regenwetter, M. and A. A. J. Marley, 2001, Random relations, random utilities and random functions. Journal of Mathematical Psychology 45:864-912.

Tversky, A. and C. R. Fox, 1995, Weighing risk and uncertainty. Psychological Review 102, 269-283.

Tversky, A. and D. Kahneman, 1992, Advances in prospect theory: Cumulative representation of uncertainty. Journal of Risk and Uncertainty 5, 297-323.

Vieider, F. M., M. Lefebvre, R. Bouchouicha, T. Chmura, R. Hakimov, M. Krawczyk and P. Martinsson, 2015, Common components of risk and uncertainty attitudes across contexts and domains: Evidence from 30 countries. Journal of the European Economic Association 13, 421-452.

von Winterfeldt, D., N.-K. Chung, R. D. Luce, and Y.-H. Cho, 1997, Tests of consequence monotonicity in decision making under uncertainty. Journal of Experimental Psychology: Learning, Memory, and Cognition, 23, 406-426.

Wakker, P., 2010, Prospect Theory: For Risk and Ambiguity. Cambridge, UK: Cambridge University Press.

Wilcox, N., 2008, Stochastic models for binary discrete choice under risk: A critical primer and econometric comparison. In J. C. Cox and G. W. Harrison, eds., Research in Experimental Economics Vol. 12: Risk Aversion in Experiments pp. 197-292. Bingley, UK: Emerald.

Wilcox, N., 2011, 'Stochastically more risk averse:' A contextual theory of stochastic discrete choice under risk. Journal of Econometrics 162, 89-104. 
Wilcox, N., 2015. Unusual estimates of probability weighting functions. Chapman University, Economic Science Institute Working Paper \#15-10.

Wu, G. and R. Gonzalez, 1999, Nonlinear decision weights in choice under uncertainty. Management Science 45, 74-85. 


\section{Appendix: A brief Monte Carlo illustration of the problem}

Simulated data sets for this brief Monte Carlo analysis of several estimation methods are based on the experimental design of Gonzalez and Wu (1999). Certainty equivalents were elicited from their subjects for $t=1,2, \ldots, 165$ distinct two-outcome prospects $\left(p_{t}, h_{t} ; 1-p_{t}, l_{t}\right)$. These were constructed by fully crossing fifteen distinct pairs of high and low outcomes $\left(h_{t}, l_{t}\right)$ with eleven distinct probabilities $p_{t}$ of receiving the high outcome $h_{t}$ (and corresponding probabilities $1-p_{t}$ of receiving the low outcome $l_{t}$ ). The eleven probabilities are $p_{t} \in\{.01, .05, .10, .25, .40, .50, .60, .75, .90, .95, .99\}$; and the fifteen high and low outcome pairs are $\left(h_{t}, l_{t}\right) \in\{(25,0),(50,0),(75,0),(100,0),(150,0),(200,0),(400,0)$, $(800,0),(50,25),(75,50),(100,50),(150,50),(150,100),(200,100),(200,150)\}$. These same 165 prospects ( 88 simple prospects and 77 non-simple prospects) are the "input" to the simulated subjects I create in the Monte Carlo data sets. Let $Z=\{0,25,50, \ldots, 800\}$ denote the set of the nine distinct outcomes found in these 165 prospects.

Each simulated subject $s=1,2, \ldots 1000$ in the first data set is given a random EU certainty equivalent for each of the 165 prospects. Each subject $s$ is endowed with parameters $k^{s}$ and $\theta^{s}$ of the Gamma distribution p.d.f. as given in Example 1 of Section 2. The parameter $k^{s}$ is drawn once for each subject from a Lognormal distribution with mean $E(k)=0.75$ and variance $\mathrm{V}(k) \approx 0.16$. The parameter $\theta^{s}$ is then chosen (given the drawn $k^{s}$ ) so that $\mathbb{C}^{e u}\left(e^{-1} \mid k^{s}, \theta^{s}\right)=\left(1+\theta^{s}\right)^{-k^{S}}=e^{-1}$. This endows each simulated subject $s$ with a random EU c.e.f. having the fixed point $e^{-1}$, as is characteristic of the 1-parameter Prelec (1998) weighting function, but also creates heterogeneity in the degree of curvature of subjects' c.e.f.s. Then for each subject $s, t=1,2, \ldots, 165$ values $x_{t}^{s}$ are independently drawn from the Gamma distribution with that subject's parameters $k^{s}$ and $\theta^{s}$. These create the 165 simulated elicited certainty equivalents $c e_{t}^{s}=\left[p_{t} h_{t}{ }^{1 / x_{t}^{s}}+\left(1-p_{t}\right) l_{t}{ }^{1 / x_{t}^{s}}\right]^{x_{t}^{s}}$ for each subject $s$. Repeating this 1000 times yields the "random EU" data set.

For comparison, I create a second data set of 1000 simulated subjects who are given standard EU certainty equivalents for each of the 165 prospects. Each simulated subject $s$ is endowed with a fixed value $x^{s}$, drawn once for each subject from a Gamma distribution with the parameters $k=0.75$ and $\theta=2.79$. For each subject $s$, this $x^{s}$ then creates 165 
expected certainty equivalents $E\left(c e_{t}^{s}\right)=\left[p_{t} h_{t}{ }^{1 / x^{s}}+\left(1-p_{t}\right) l_{t}{ }^{1 / x^{s}}\right]^{x^{s}}:$ These are standard EU c.e.f.s, and one must somehow add standard model errors to them. To do this, notice that each expected certainty equivalent may be rewritten as a proportion of the interval $\left[l_{t}, h_{t}\right]$, that is as $\Delta_{t}^{s}=\left(E\left(c e_{t}^{s}\right)-l_{t}\right) /\left(h_{t}-l_{t}\right)$. One may then interpret this proportion as the mean of a Beta distribution on the interval $(0,1)$ and define parameters of that Beta distribution as $\alpha_{t}^{S}=\varpi \Delta_{t}^{S}$ and $\beta_{t}^{S}=\varpi\left(1-\Delta_{t}^{S}\right)$. (Beta distributions may be parameterized in terms of their mean $\Delta \in(0,1)$ and an inverse dispersion parameter $\varpi>0$, from which their more usual parameterization $\alpha \equiv \varpi \Delta$ and $\beta \equiv \varpi(1-\Delta)$ may be had. I chose $\varpi=6$ to give the resulting simulated certainty equivalents $c e_{t}^{s}$ in this simulated Standard EU data conditional variances resembling those found in the simulated Random EU data.) Then one may draw a beta variate $y_{t}^{s}$ on $(0,1)$ using these parameters, and the simulated certainty equivalents with their standard model error become $c e_{t}^{s}=l_{t}+\left(h_{t}-l_{t}\right) y_{t}^{s}$.

I consider four estimation methods. The first two methods use a standard RDU model of the c.e.f. of the $c e_{t}^{s}$, that is $E\left(c e_{t}^{s} \mid v^{s}, w^{s}\right)=\left(v^{s}\right)^{-1}\left[w^{s}\left(p_{t}\right) v^{s}\left(h_{t}\right)+\left(1-w^{s}\left(p_{t}\right)\right) v^{s}\left(l_{t}\right)\right]$; the corresponding empirical model is then $c e_{t}^{s}=E\left(c e_{t}^{s} \mid v^{s}, w^{s}\right)+\varepsilon_{t}^{s}$. I make the standard assumptions about the error, those being $E\left(\varepsilon_{t}^{s}\right)=E\left(\varepsilon_{t}^{s} \mid p_{t}, h_{t}, l_{t}\right)=0$, but also adopt the assumption of Bruhin, Fehr-Duda and Epper (2010) that $\operatorname{Var}\left(\varepsilon_{t}^{S}\right)$ is proportional to $\left(h_{t}-l_{t}\right)^{2}$ for each subject. (This assumption happens to be true for the simulated Standard EU data.) This implies a "weighted error" $\epsilon_{t}^{s}=\left[c e_{t}^{s}-E\left(c e_{t}^{s} \mid v^{s}, w^{s}\right)\right] /\left(h_{t}-l_{t}\right)$, and the first two estimation methods optimize a function of these weighted errors.

The first estimation method combines a nonlinear least squares estimator with lean 1parameter forms of the functions $w^{s}$ and $v^{s}, w^{s}(p)=p^{\gamma^{s}} /\left[p^{\gamma^{s}}+(1-p)^{\gamma^{s}}\right]^{1 / \gamma^{s}}$ and $v^{s}(z)=z^{\sigma^{s}}$. This is the estimation method of Tversky and Kahneman (1992): I'll call it NLS-M-L (for “nonlinear least squares, money errors, lean parameterization”). The second estimation method combines a maximum likelihood estimator with the same $v^{s}(z)=z^{\sigma^{s}}$, but a more expansive 2-parameter weighting function $w^{s}(q)=\delta^{s} p^{\gamma^{s}} /\left[\delta^{s} p^{\gamma^{s}}+(1-p)^{\gamma^{s}}\right]$. The weighted error $\epsilon_{t}^{S}$ is assumed to have a Normal distribution with zero mean and constant variance. This estimation method is inspired by Bruhin, Fehr-Duda and Epper (2010), but I will always estimate at the individual subject level whereas they estimated 
finite mixture models of the subject population and included prospect-specific error variance terms (which cannot be done in the case of subject-level estimation). I'll call this method ML-M-C (for "maximum likelihood, money errors, common parameterization"). The power utility function, combined with some 2-parameter weighting function, is quite common in the literature on risk preference estimation.

The third method writes an estimating equation in utility rather than money terms, and the parameterizations of $v^{s}$ and $w^{s}$ are maximally expansive. There are nine distinct outcomes in $Z$, so there are nine distinct values of $v^{s}(z)$. Since the RDU value function is an interval scale, one can choose $v^{s}(0)=0$ and $v^{s}(800)=1$, leaving seven unique and distinct values of $v^{s}(z)$ as seven parameters to estimate. Similarly, the eleven distinct probabilities in the experiment become eleven distinct parameters $w^{s}\left(p_{t}\right)$ to estimate. Now linearly interpolate $v^{S}\left(c e_{t}^{S}\right)$ from the parameters $v^{s}(z)$ in the following manner. Let $l u b\left(c e_{t}^{S}\right)=\min _{z \in Z} z \mid z \geq c e_{t}^{S}$ and $g l b\left(c e_{t}^{S}\right)=\max _{z \in Z} z \mid z \leq c e_{t}^{S}$ be the least upper bound and greatest lower bound (among the nine outcomes in the experiment) on $c e_{t}^{s}$, with values given by the parameter values $v^{S}\left(l u b\left(c e_{t}^{S}\right)\right)$ and $v^{s}\left(g l b\left(c e_{t}^{S}\right)\right)$. Then define

$$
\tilde{v}^{S}\left(c e_{t}^{S}\right)=\frac{\left[l u b\left(c e_{t}^{S}\right)-c e_{t}^{S}\right] v^{s}\left(g l b\left(c e_{t}^{S}\right)\right)+\left[c e_{t}^{S}-g l b\left(c e_{t}^{S}\right)\right] v^{s}\left(l u b\left(c e_{t}^{S}\right)\right)}{l u b\left(c e_{t}^{S}\right)-g l b\left(c e_{t}^{S}\right)},
$$

a linear interpolation of $v^{s}\left(c e_{t}^{S}\right)$. This estimation method then assumes that the c.e.f. of $\tilde{v}^{s}\left(C_{t}^{S}\right)$ is the RDU of prospect $t$, that is $E\left(\tilde{v}^{s}\left(c e_{t}^{S}\right) \mid v^{s}, w^{s}\right)=w^{s}\left(p_{t}\right) v^{s}\left(h_{t}\right)+(1-$ $\left.w^{s}\left(p_{t}\right)\right) v^{s}\left(l_{t}\right)$, and one may then think of $\tilde{v}^{s}\left(c e_{t}^{s}\right)-E\left(\tilde{v}^{s}\left(c e_{t}^{s}\right) \mid v^{s}, w^{s}\right)$ as a "utility error." Following Wilcox (2011), assume the variance of these utility errors is proportional to $\left[v^{s}\left(h_{t}\right)-v^{s}\left(l_{t}\right)\right]^{2}$. Then $\zeta_{t}^{s}=\left[\tilde{v}^{s}\left(C_{t}^{s}\right)-E\left(\tilde{v}^{s}\left(C_{t}^{s}\right) \mid v^{s}, w^{s}\right)\right] /\left[v^{s}\left(h_{t}\right)-v^{s}\left(l_{t}\right)\right]$ is a weighted utility error that becomes the object of nonlinear least squares estimation. I call this the NLS-U-E estimation (for "ㅁonlinear least squares, utility errors, expansive parameterization"). It is inspired by Gonzalez and Wu's (1999) estimation method, though there are several differences between their method and this one (see Gonzalez and Wu 1999, pp.146-148, for details).

Finally, I consider an estimation method that may sidestep the issue identified in the text. Rather than taking $\left(v^{s}\right)^{-1}\left[w^{s}\left(p_{t}\right) v^{s}\left(h_{t}\right)+\left(1-w^{s}\left(p_{t}\right)\right) v^{s}\left(l_{t}\right)\right]$ to be the conditional mean of $c e_{t}^{S}$, this last estimation method takes this to be the conditional median of $c e_{t}^{S}$ : 
That is, let $\operatorname{Med}\left(c e_{t}^{S} \mid v^{s}, w^{s}\right)=\left(v^{s}\right)^{-1}\left[w^{s}\left(p_{t}\right) v^{s}\left(h_{t}\right)+\left(1-w^{s}\left(p_{t}\right)\right) v^{s}\left(l_{t}\right)\right]$, and let weighted money errors be $\epsilon_{t}^{S}=\left[C_{t}^{S}-\operatorname{Med}\left(C_{t}^{S} \mid v^{s}, w^{s}\right)\right] /\left(h_{t}-l_{t}\right)$. Although these errors have exactly the same form as the errors in the first two methods, the fact that we wish to estimate a conditional median function (rather than a c.e.f.) implies that least squares is not the appropriate estimator: Rather, we want a least absolute deviation or LAD estimator. Combined with the same lean parameterization used for the first method, I call this the LAD-M-L estimation (for "least absolute deviation, money errors, lean parameterization").

With the exception of the NLS-U-E estimation method, the well-known simplex algorithm of Nelder and Mead (1965) was used to optimize objective functions. For the NLS-U-E estimation method, I imposed monotonicity constraints on the estimated $v^{s}(z)$ and $w^{s}\left(p_{t}\right)$ (one difference versus Gonzalez and $\mathrm{Wu} 1999$ ) and this requires a different optimization algorithm: Powell's (1992) COBYLA algorithm is used for this estimation instead. All estimations were performed using the SAS procedure "NLP" (nonlinear programming) in the SAS 9.4 version of the SAS/OR software.

Rather than providing tabular results of these four estimation methods as applied to the two data sets, I provide a sequence of eight figures. The features of each figure are identical. Estimated weighting functions for the first 250 subjects in each data set are plotted as quite thin, light greyscale lines on a black background: This has the effect of representing the behavior of each method as a light cloud of lines. A heavy light grey identity line shows the (linear, identity) weighting function of an EU subject; deviations from this line represent both sampling variability and possible bias in the estimations. Finally, a heavy dashed white line plots the mean estimated probability weight (across all 1000 subjects in each simulated data set) at each of the eleven values of $p_{t}$ in the experimental design: Since all simulated subjects in both data sets are EU subjects with identity weighting functions, deviations of this heavy dashed white line from the identity line illustrate the bias of each estimation method in each data set.

The figures come in pairs on each page that follows. Each page presents the results for one estimation method, with the top and bottom figures showing results for the Standard EU and Random EU data sets, respectively. The pair of Figures A1-a and A1-b show results for the NLS-M-L estimation method; Figures A2-a and A2-b show results for the ML-M-C 
method; Figures A3-a and A3-b show results for the NLS-U-E method; and Figures A4-a and A4-b show results for the LAD-M-L method.

None of these four estimation methods are bias-free for both the Standard EU and Random EU data sets, and this is the primary finding of this appendix. The method NLS-U-E is biased towards finding inverse-s probability weighting for both data sets: In the case of the Standard EU data I suspect this is because this method is just too parametrically expansive for the sample size. By contrast, the NLS-M-L and ML-M-C methods are virtually unbiased for Standard EU data, while they show the predicted bias when applied to the Random EU data. As speculated, the LAD-M-L method provides unbiased (and astonishingly tight) estimates for the Random EU data, but displays a quite noticeable bias in the Standard EU data in a direction opposite to inverse-s probability weighting. The latter finding (unexpected by me) may occur because the standard EU errors are drawn from Beta distributions: Though those errors have a zero mean by construction, beta distributions are generally skewed so that these errors would not usually have zero median (as required for proper LAD estimation). In sum, none of these four estimation methods are robust to the underlying source of randomness in the data generating process. 

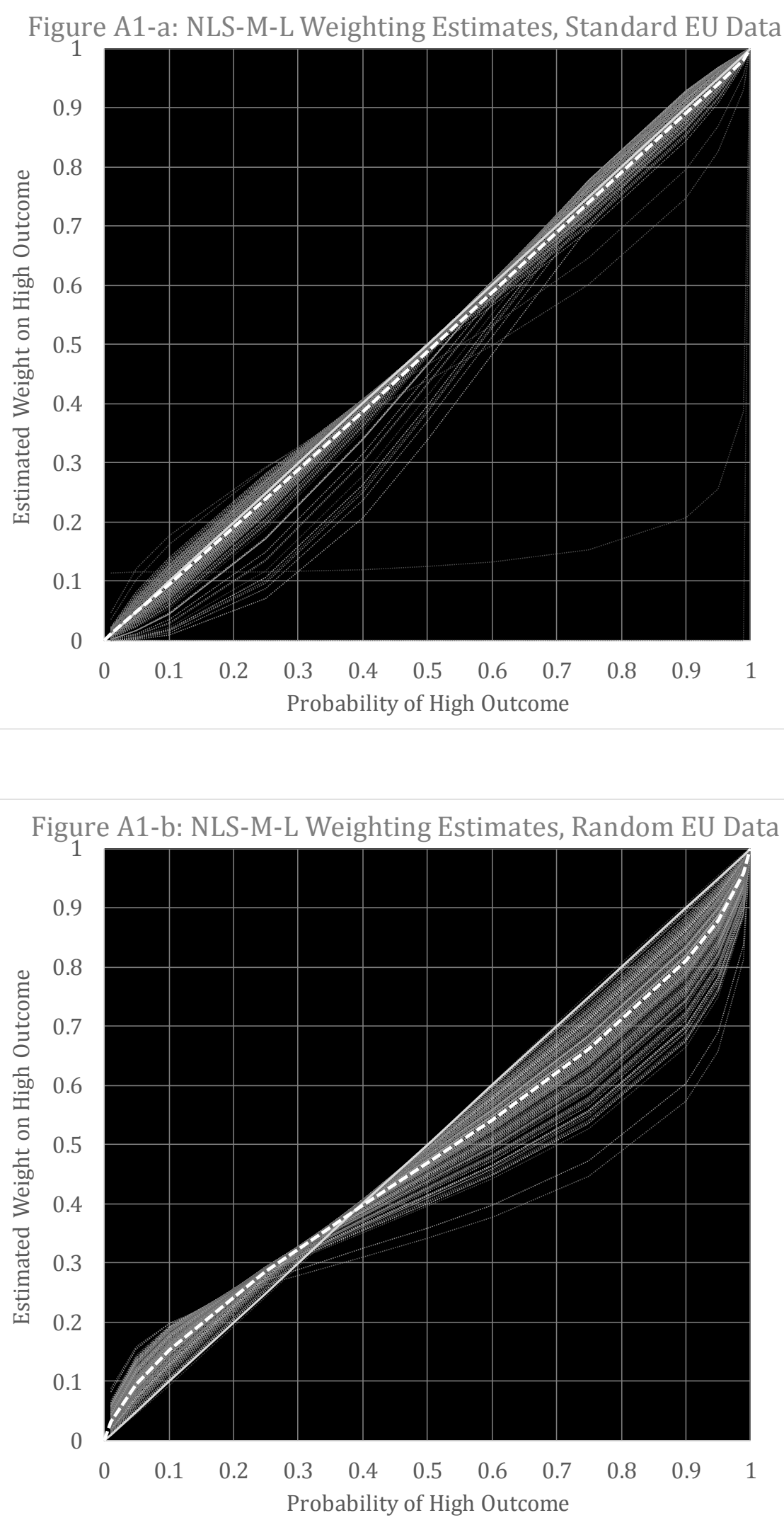

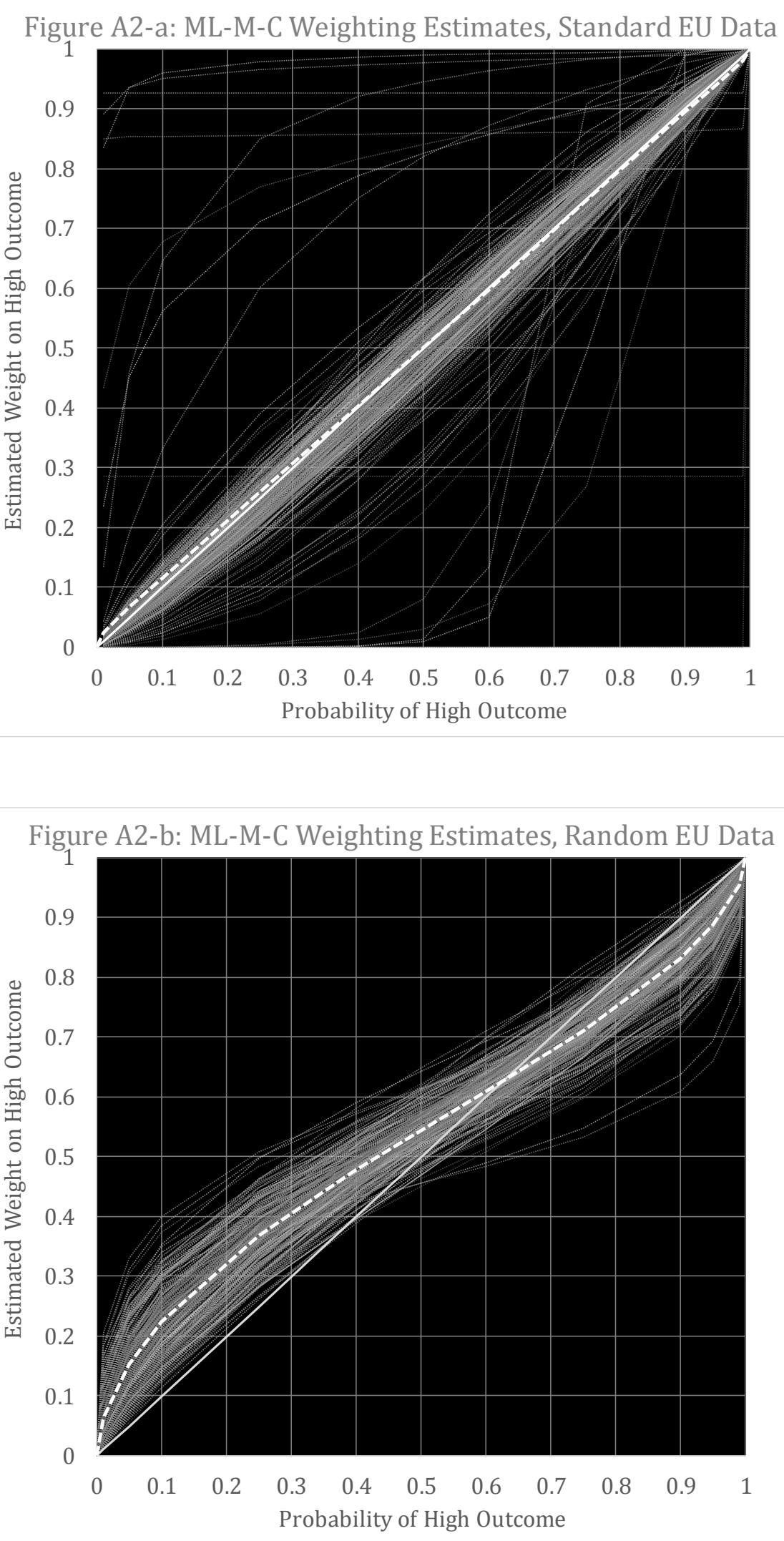

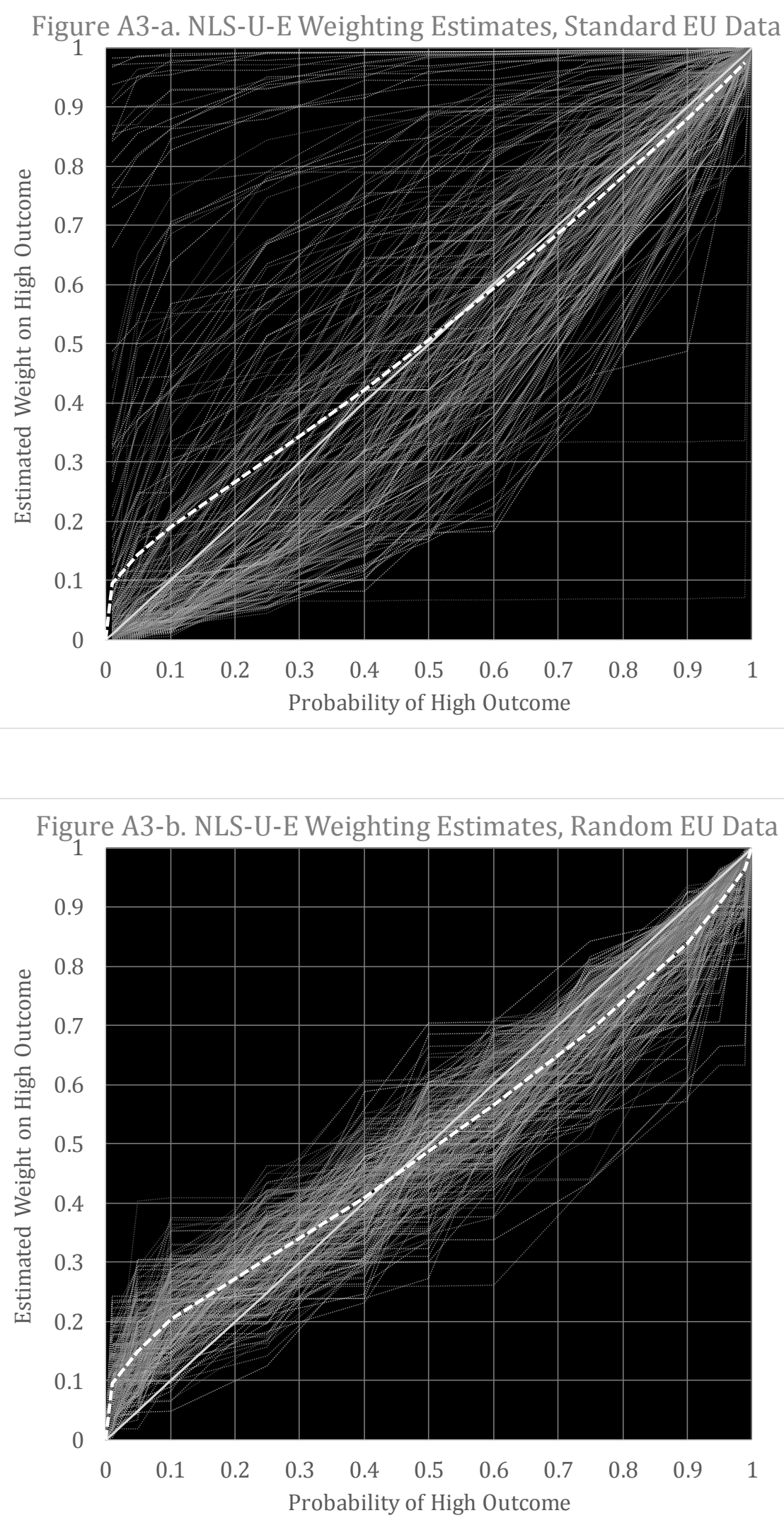

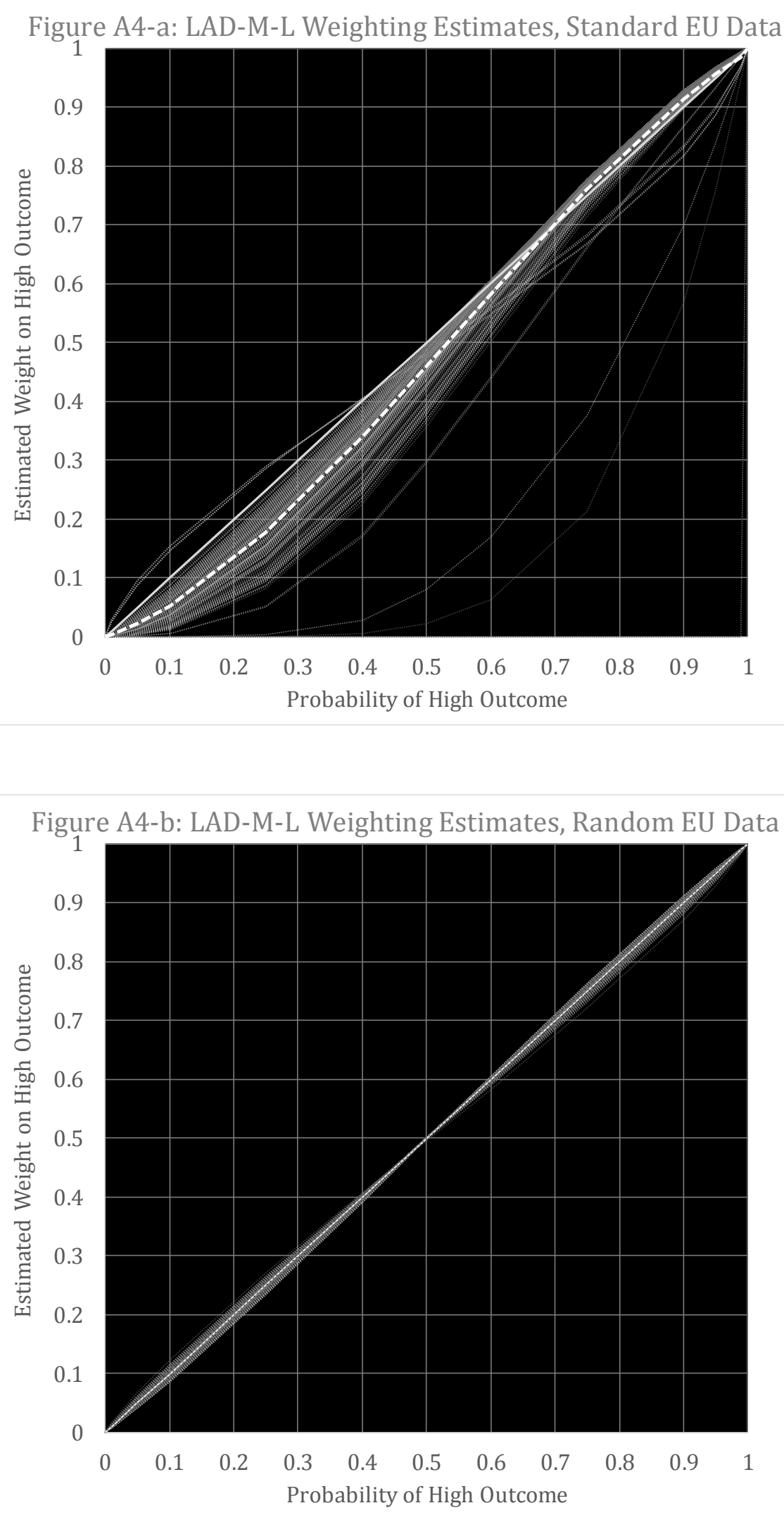